\title{
Cardiac magnetic resonance imaging in congenital heart disease
}

\author{
Tobias Rutz ${ }^{\mathrm{a}^{*}}$, Kerstin Wustmann ${ }^{\mathrm{b} *}$, Milan Prsa ${ }^{c}$, Jean-Paul Vallée ${ }^{\mathrm{d}}$, Birgit Donner ${ }^{\mathrm{e}}$, Jens Bremerich ${ }^{\mathrm{f}}$, \\ Christina Deluigi ${ }^{b}$, Michael Zellweger ${ }^{g}$, Daniel Toblerg, Christian Kellenberger ${ }^{h}$, Andreas Wahlb, \\ Pierre Monneya ${ }^{\text {, Peter Buser }}{ }^{9}$, François Gudinchet ${ }^{i}$, Jürg Schwitter ${ }^{a}$, Emanuela Valsangiacomo Büchel ${ }^{\mathrm{j}}$
}

a Division of Cardiology and Cardiac MR Centre, Centre Hospitalier Universitaire Vaudois, CHUV, Lausanne, Switzerland; 'b Department of Cardiology, Inselspital, University Hospital Bern, Switzerland; ' Division of Cardiology, Department of Paediatrics, University Hospital of Lausanne, CHUV, Lausanne, Switzerland; ' Service of Radiology, University Hospitals of Geneva, Switzerland; ${ }^{e}$ Division of Paediatric Cardiology, University Children's Hospital, UKBB, University of Basel, Switzerland; ${ }^{\dagger}$ Division of Radiology, University Hospital Basel, Switzerland; ${ }^{9}$ Division of Cardiology, University Hospital Basel, Switzerland; h Division of Radiology, University Children's Hospital, Zurich, Switzerland; i Paediatric Radiology Unit, Department of Radiology, University Hospital of Lausanne, CHUV, Lausanne, Switzerland; ' Division of Paediatric Cardiology, Paediatric Heart Centre, University Children's Hospital, Zurich, Switzerland * TR and KW contributed equally to the document

Position paper of a group of Swiss paediatric and adult cardiologists, and radiologists performing cardiac magnetic resonance in congenital heart disease, endorsed by the working groups "Adult Congenital Heart Disease" (WATCH) and "Echocardiography and Cardiac Imaging" of the Swiss Society of Cardiology, the Swiss Society of Pediatric Cardiology and the Ressort Cardiac Imaging of the Swiss Society of Radiology

\section{Introduction}

This document has been written by paediatric and adult cardiologists as well as radiologists with expertise in the field of congenital heart disease (CHD) imaging from the five university hospitals in Switzerland. Cardiac magnetic resonance (CMR) is one of the basic imaging modalities for assessment of CHD, with increasing use. This document aims to harmonise imaging protocols and seeks high quality and comparability of CMR examinations between centres treating paediatric and adult CHD patients in Switzerland. Comparable image quality will simplify scientific collaboration and facilitate designing prospective studies. The document summarises in a first part (sections 2 to 5) general and technical aspects of CMR in CHD. The second part (section 6) provides protocols for the most common congenital heart defects, based on current guidelines and position papers [1-4]. We define for every lesion the standard for a comprehensive CMR examination by describing the mandatory and optional sequences that should be acquired. However, every centre is free to expand the protocols according to patients' individual clinical situations and indications, as well as to address centre-specific issues. This document does not replace standard CMR textbooks or international guidelines published by, for example, the Society of Cardiac Magnetic Resonance or the European Society of Cardiology [1-3].

\section{Aspects specific to infants and small children}

In children younger than 7 years of age, CMR usually needs to be performed under sedation or general anaesthesia. However, in neonates and infants under 6 months of age, a successful examination can also be performed without sedation or general anaesthesia, by using the "feed and wrap" technique [5]. CMR in infants

\footnotetext{
Abbreviations:

AV = atrioventricular

$\mathrm{BCPC}=$ bidirectional cavopulmonary connection

CE MRA = contrast-enhanced magnetic resonance angiography

CHD = congenital heart disease

$\mathrm{CMR}=$ cardiac magnetic resonance

GBCA = gadolinium-based contrast agent

ICD = implantable cardioverter-defibrillator

IVC = inferior vena cava

LGE = late gadolinium enhancement

LPA = left pulmonary artery

MPA = main pulmonary artery

$\mathrm{LV} \quad=$ left ventricle

OT = outflow tract

PDA = patent ductus arteriosus

$\mathrm{PR} \quad=$ pulmonary regurgitation

$\mathrm{PC} C M R=$ phase contrast cardiac magnetic resonance

$\mathrm{PA} \quad=$ pulmonary artery

$\mathrm{PR} \quad=$ pulmonary regurgitation

$\mathrm{Qp} / \mathrm{Qs}=$ ratio of pulmonary to systemic blood flow

RPA = right pulmonary artery

RV = right ventricle

SVC = superior vena cava

SSFP = steady-state free precession

TCPC = total cavopulmonary connection

TGA = transposition of the great arteries

VSD $\quad=$ ventricular septal defect
} 
and small children poses technical challenges because of the small size of the structures and rapid heart rates, which require sequence optimisation [6]. High spatial resolution can be achieved by using thin slices, a small field of view and a large matrix. To maintain a high signal to noise ratio, smaller multi-element coils and multiple signal averages are often necessary. Achieving a high temporal resolution requires decreasing the number of views per segment. For contrast-enhanced magnetic resonance angiography (CE MRA) in infants, the small volumes of contrast used are best injected by hand (injection during $80 \%$ of scan time).

\section{Safety aspects}

\section{General}

Common contraindications for CMR must be respected (claustrophobia, non-CMR compatible neurostimulators, insulin pumps, epidural pumps, etc.).

\section{Gadolinium-based contrast agents}

Gadolinium-based contrast agents (GBCAs) are generally safe; however, a few restrictions and contraindications exist and must be respected [7]. Major adverse events are very rare, and consist of allergic reactions and nephrogenic systemic fibrosis [7]. Nephrogenic systemic fibrosis is an exceedingly rarely reported generalised disease. Risk is increased in patients with severely decreased renal function and a glomerular filtration rate below $30 \mathrm{ml} / \mathrm{min}$. More recently, deposition of gadolinium in the brain has been reported in patients who underwent repeated magnetic resonance imaging (more than five times) for various (noncardiac) indications; the clinical significance of this finding is not known yet. In general, if administration of GBCAs is required, such as for angiography, perfusion studies or late gadolinium enhancement, the use of nonlinear, cyclic GBCAs is preferred, as for this group of contrast agents no or very few cases of nephrogenic systemic fibrosis have been reported and brain deposition may be less [8-11]. In patients with a glomerular filtration rate below $30 \mathrm{ml} / \mathrm{min}$, the use of a cyclic GBCA should be carefully weighed against the potential risks and, if possible, avoided, while some linear GBCAs are contraindicated [12]. If the use of GBCAs is definitely contraindicated, then 3D steady-state free precession (SSFP) sequences are a valuable alternative, obviating the need for CE MRA.

Administration of GBCAs to children below 2 years of age is currently off-label use and only cyclic GBCAs should be used.

\section{Pacemakers, implantable cardioverter-} defibrillators, epicardial pacemaker leads

CMR is generally contraindicated in patients after pacemaker or implantable cardioverter-defibrillator (ICD) implantation. However, several implantable electrodes and generators of the newer generations are CMR conditional; performing CMR examinations with these devices is safe and yields high-quality images [13]. Precautions and limitations of the implanted devices and electrodes must be respected $[14,15]$. The presence of epicardial pacemaker leads is still a contraindication for CMR because of potential heating of the leads. In patients who have undergone cardiac surgery, a chest X-ray is recommended after surgery and/or before the CMR examination to exclude the presence of residual epicardial pacemaker leads.

\section{Pregnancy, breast-feeding}

The risks and benefits of any imaging modality in pregnant patients must be balanced. CMR with maximum field strength of $1.5 \mathrm{~T}$ is considered safe regardless of gestational age when the benefit outweighs the risks; however, if possible it should be postponed until after the first trimester [16]. CMR should be preferred to radioactive imaging modalities [17]. The use of GBCAs in pregnancy should be avoided as they cross the blood-placenta barrier and their effects on the fetus are not known $[16,18]$. Only in clinical situations that do not allow the required information to be obtained without contrast agents, the use of GBCAs can be discussed, and the lowest possible dose should be administered.

A very small percentage of the GBCA dose, which is much lower than the maximum permitted intravenous dose for infants, is excreted in breast milk after intravenous administration to breast-feeding patients [19]. Nevertheless, interruption of breastfeeding for 24 hours after exposure to a GBCA is recommended.

\section{Myocardial stress perfusion examinations}

A medical doctor with training in cardiopulmonary resuscitation must be present during myocardial perfusion studies with adenosine, dipyridamole or dobutamine. Medication for cardiopulmonary life support and an external cardioverter-defibrillator must be available outside the scanning room and be ready for use.

\section{Medical devices and implants other than pacemakers and ICDs}

Respect the recommendations for use of the different implantable medical devices when performing CMR. 


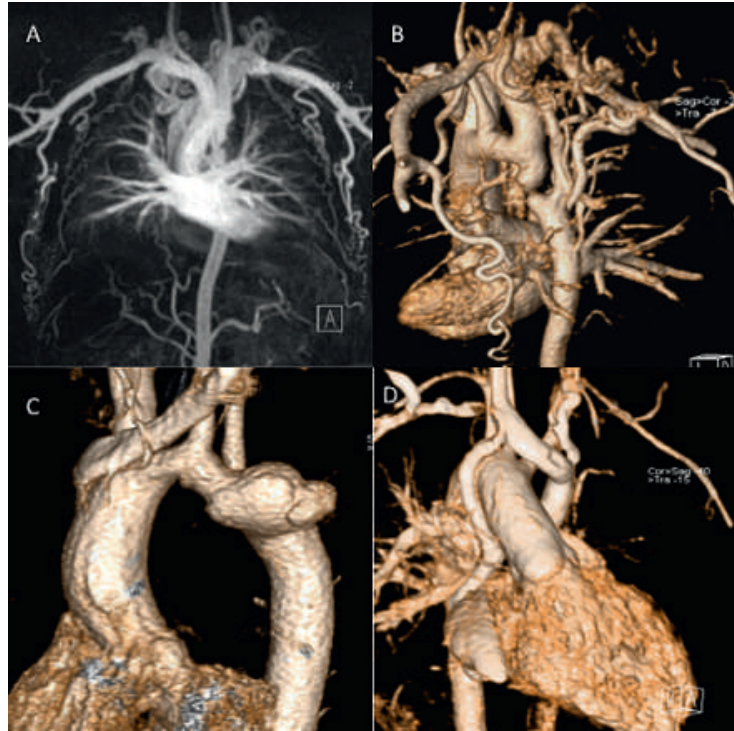

Figure 1: Contrast-enhanced CMR angiography. Severe coarctation with large collaterals. The descending aorta is almost interrupted ( $\mathbf{A}$ and $\mathbf{B}$ ). Hypoplastic aortic arch and large pseudoaneurysm 28 years after patch repair for aortic coarctation (C).

Patient from figure 1 with situs inversus and univentricular heart and TCPC. A persistant right superior vena cava (SVC) is still connected to the atrium and accounts for a $R-L$ shunt (D).

Table 1: Technical details of steady-state free precession.

\begin{tabular}{|c|c|c|}
\hline & $<10 \mathrm{~kg}$ & $\geq 10 \mathrm{~kg}$ \\
\hline In-plane resolution $(\mathrm{mm})$ & $1.0-1.5$ & $1.3-2.0$ \\
\hline Slice thickness (mm) & $3-7$ & $6-8$ \\
\hline Temporal resolution & $25-50 \mathrm{~ms}$ (or $4-5 \%$ of RR interval) & $35-50 \mathrm{~ms}$ \\
\hline Number of signal averages & $\begin{array}{l}1-3 \text { (higher signal averages if free } \\
\text { breathing in small children) }\end{array}$ & $1-2$ \\
\hline Respiratory compensation & Free breathing & Breath-hold \\
\hline Interslice gap (mm) & $0-2$ & $0-2$ \\
\hline Reconstructed phases & $25-40$ & $25-40$ \\
\hline ECG gating & \multicolumn{2}{|c|}{ Retrospective or prospective if arrhythmia } \\
\hline
\end{tabular}

Table 2: Technical details of contrast-enhanced magentic resonance angiography.

\begin{tabular}{|c|c|c|c|}
\hline & $<10 \mathrm{~kg}$ & & $\geq 10 \mathrm{~kg}$ \\
\hline In-plane resolution $(\mathrm{mm}) *$ & $\leq 1.2 \times \leq 1.2$ & & $\leq 1.5 \times \leq 1.5$ \\
\hline Slice thickness (mm) & $\leq 1.2$ & & $\leq 2$ \\
\hline Respiratory compensation & Free breathing & Breath-hold & Breath-hold \\
\hline
\end{tabular}

Table 3: Technical details 3D whole heart imaging, with or without navigator technique.

\begin{tabular}{lll}
\hline & $<10 \mathbf{~ k g}$ & $\geq 10 \mathbf{~ k g}$ \\
\hline Isotropic resolution $\left(\mathbf{m m}^{3}\right)$ & $1.2-1.5$ & $1.3-2.0$ \\
\hline Navigator window $(\mathbf{m m})$ & 3 & 5 \\
\hline Image acquisition window $(\mathbf{m s})$ & $40-60$ & $80-150$ \\
\hline Trigger delay & Endsystole or mid-diastole & Mid-diastole \\
\hline
\end{tabular}

Information about product safety is available on dedicated websites (e.g., www.mrisafety.com).

\section{Technical aspects}

The imaging parameters given in the tables represent the consensus among the different centres and are in agreement with current recommendations [1-3].

\section{Field strength}

Current recommended field strengths are 1.5 or $3 \mathrm{~T}$.

\section{Sequences}

\section{Visualisation of anatomy}

Sequences: SSFP, spin echo, fast spin echo.

\section{Cine imaging}

Sequences: SSFP, standard spoiled gradient echo pulse sequence (technical details see table 1 ).

\section{Contrast-enhanced magnetic resonance angiog- raphy}

A GBCA dose of $0.1-0.2 \mathrm{mmol} / \mathrm{kg}$ is injected as a bolus. In children, administration during $80 \%$ of the image acquisition time is proposed. Image acquisition should, if possible, be during breath holding; timing of acquisition depends on the vascular structure to be visualised. Depending on indication, consider timeresolved CE MRA (for example see fig. 1), for technical details see table 2 .

\section{D whole heart (SSFP)}

Whole heart imaging is performed using 3D SSFP, either ECG triggered and respiratory navigator gated during free breathing, or self-navigation 3D approaches with isotropic resolution (for resolution see table 3) [20-22]. Acquisition volume is adapted to patient collaboration and targeted structures to be visualised in order to minimise scan time and optimise quality.

\section{Ventricular volumetry}

Acquisition: We recommend acquiring a short-axis stack of contiguous slices covering both ventricles from base to apex, with the first and last slices without ventricular mass to ensure a complete coverage of the ventricles (figs $2 \mathrm{a}$ and $2 \mathrm{~b}$ ). For dilated and hypertrophied right ventricles, a separate stack oriented parallel to the tricuspid valve plane to assure optimal alignment should be considered. Additionally, a transaxial stack or long-axis stack can be considered in specific lesions. Details of cine imaging according to patient size are described in table 1. 


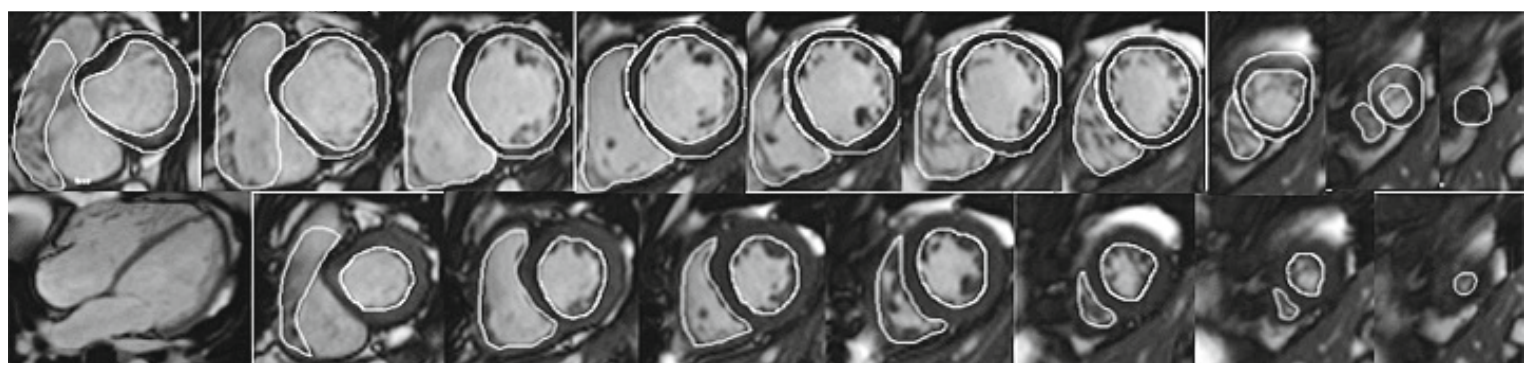

Figure 2a: SSFP short axis cine stack with volumetry of both ventricles. Top: Tracings of the RV and LV endocardial borders and LV epicardial border. Bottom: SSFP end-diastolic still frame of 4-chamber view. Systolic tracings of the RV and LV endocardial borders. LV ejection fraction (EF) 61\%, LV end-diastolic volume index (EDVi) $94 \mathrm{ml} / \mathrm{m}^{2}$, LV endsystolic volume index (ESVi) $37 \mathrm{ml} /$ $\mathrm{m}^{2}$, LV mass index $67 \mathrm{~g} / \mathrm{m}^{2}$. RVEF 61\%, RVEDVi $80 \mathrm{ml} / \mathrm{m}^{2}$, RVESVi $31 \mathrm{ml} / \mathrm{m}^{2}$. Patient with surgically repaired patent ductus arteriosus and correction of coronary anomaly (left anterior descending artery originating from MPA), mild aortic regurgitation.

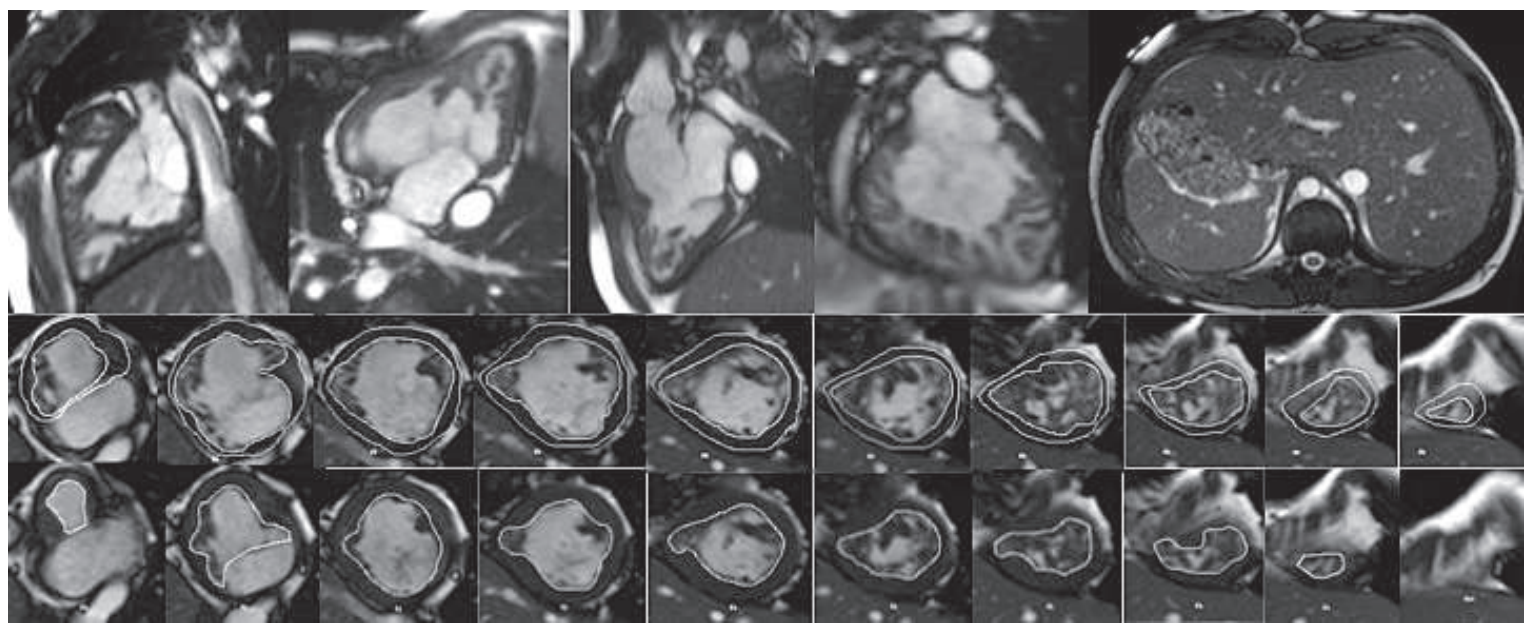

Figure 2b: Univentricular heart with situs inversus, mesocardia, unbalanced atrioventricular (AV) septal defect with common AV valve, right ventricular dominance, transposition and total cavopulmonary connection (TCPC).

Top: SSFP long axis cine still frames and abdominal situs.

Bottom: SSFP short axis cine stack with volumetry of both ventricles together: VEDVi $125 \mathrm{ml} / \mathrm{m}^{2}$, EF 42\%.

Analysis: The use of dedicated software is recommended. Endo- and epicardial left and right ventricular borders are manually traced at end-diastole and end-systole. The papillary muscles are included in the blood pool. The interventricular septum is included in the left ventricular (LV) mass. Practice varies regarding whether the right ventricular (RV) trabeculations are

Table 4: Technical details of phase contrast CMR.

\begin{tabular}{lll}
\hline & $<\mathbf{1 0 ~} \mathbf{~ g}$ & $\geq \mathbf{1 0} \mathbf{~ k g}$ \\
\hline In-plane resolution (mm) & $1.0-1.2$ & $1.0-2.0$ \\
\hline Slice thickness (mm) & $4-5$ & $4-6$ \\
\hline Temporal resolution & $\begin{array}{l}25-50 \mathrm{~ms} \text { or } 5 \% \\
\text { of RR interval }\end{array}$ & $\begin{array}{l}25-50 \mathrm{~ms} \text { or } 5 \% \\
\text { of RR interval }\end{array}$ \\
\hline Number of signal averages & $1-3$ & \\
\hline Reconstructed phases per RR interval & $20-30$ & \\
\hline Velocity encoding (cm/s) & $\begin{array}{l}\text { To be set according to expected maximum } \\
\text { velocities (under normal conditions: artery } \\
150 \mathrm{~cm} / \mathrm{s}, \text { vein } 80-100 \mathrm{~cm} / \mathrm{s}, \text { AV valve inflow } \\
150 \mathrm{~cm} / \mathrm{s} \text { ) }\end{array}$ \\
\hline Cardiac/respiratory motion & $\begin{array}{l}\text { Retrospective ECG gating, with or without } \\
\text { breath-hold (see text) }\end{array}$ \\
\hline
\end{tabular}

excluded from or included in the blood pool. However, higher reproducibility has been reported for segmentation along the compacted myocardium and inclusion of trabeculations in the blood pool [23]. In the case of bundle-branch block, the end-diastolic and endsystolic phases of the right and left ventricles may occur at different times; thus the phase with the largest/smallest ventricular volume has to be evaluated for each ventricle independently. The RV outflow tract (OT) should be considered part of the right ventricle, even in cases with aneurysmatic dilatation such as in patients with repaired tetralogy of Fallot. Contour data for volumetric analysis should be stored in a database and remain available for comparison the time of a subsequent examination $[1,2]$.

\section{Flow measurements by phase contrast CMR}

Acquisition: Phase contrast (PC) CMR images are usually acquired strictly perpendicular to the course of the vessel and tailored with use two orthogonal planning views (double oblique). The region of interest 


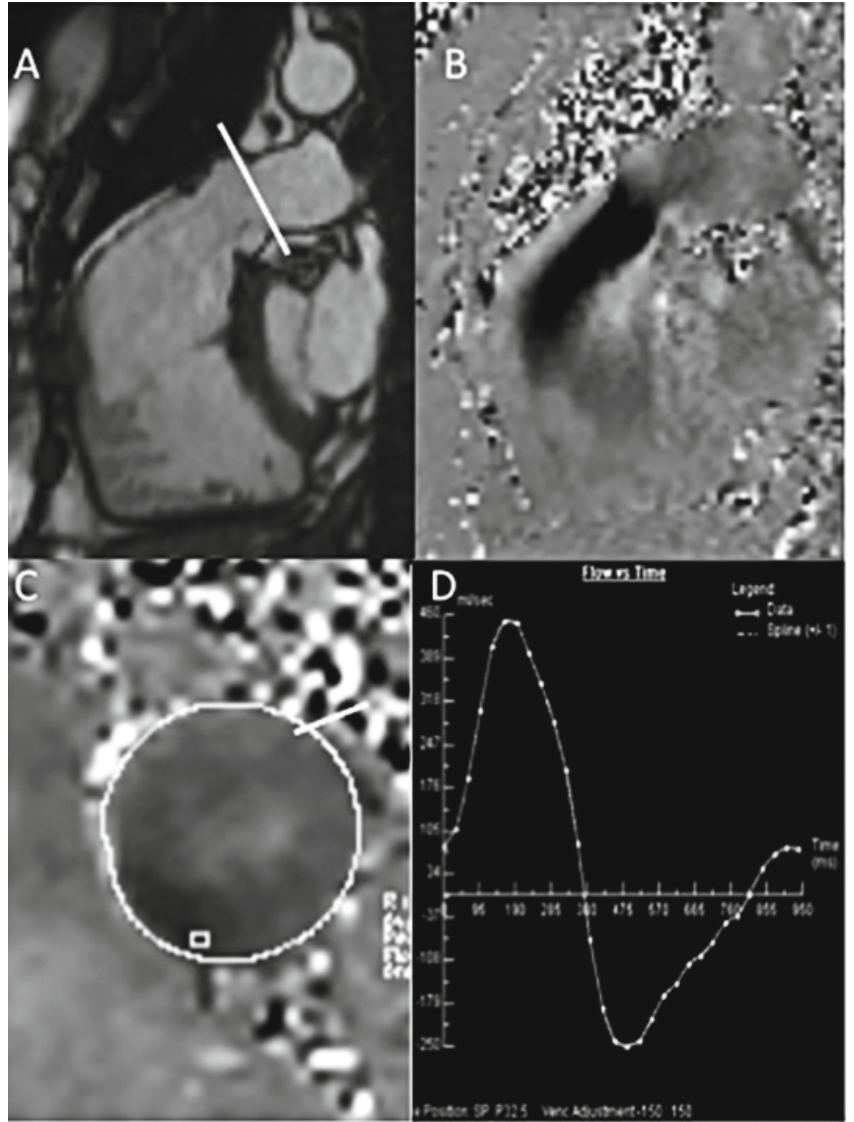

Figure 3a: A patient with transannular patch repair and ventricular septal defect (VSD) closure for tetralogy of Fallot and residual pulmonary regurgitation (PR). Still frame of a SSFP RVOT cine (A), inplane PC CMR RVOT with diastolic regurgitant jet in black (B), throughplane PC in the MPA (C), analysis showing severe PR, regurgitant volume $52 \mathrm{ml}$, regurgitant fraction $40 \%$ (D).

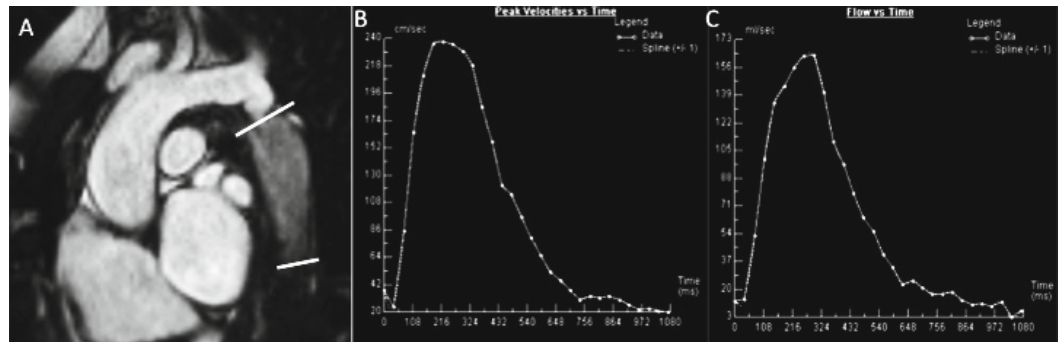

Figure 3b: Native coarctation. Throughplane PC CMR. SSFP still frame showing levels of PC CMR measurements (A). Flow curve of measurement at the level of coarctation (C-D). Peak velocity across the descending aorta $2.43 \mathrm{~m} / \mathrm{s}$ (beware of underestimation by CMR) (C), flow curve of measurement of descending aorta at the level of diaphragm with visualisation of the diastolic run-off (D).

Table 5: Technical details myocardial stress perfusion.

\begin{tabular}{lll}
\hline & $<10 \mathbf{~ k g}$ & $\geq 10 \mathbf{~ k g}$ \\
\hline In-plane resolution $(\mathbf{m m})$ & $1.5-2.0$ & $1.5-2.5$ \\
\hline Slice thickness $(\mathbf{m m})$ & $5-8$ & $8-10$ \\
\hline Acquisition of short-axis stack & Every 1 RR or 2 RR & Every 1 RR or 2 RR \\
\hline Acquisition window & $<150 \mathrm{~ms}$ & $<150 \mathrm{~ms}$ \\
\hline Signal increase (\% of baseline signal) & $>250-300 \%$ & $>250-300 \%$ \\
\hline
\end{tabular}

should be positioned in the isocentre of the scanner (figs 3a and 3b) [24].

Velocity encoding of approximately $120 \%$ of expected maximum velocity is used to optimise the dynamic range and assure absence of aliasing. Breath-hold acquisitions are associated with fewer motion artifacts. Non-breath-hold acquisitions are suggested in the Fontan circulation and for venous flow measurements, or when higher temporal and spatial resolution are required [25]. In the case of turbulent flow in the main pulmonary artery (MPA), flow measurements in the right and left branch pulmonary arteries or in each pulmonary vein to calculate pulmonary stroke volume are recommended. In the case of turbulent flow in the ascending aorta, try to perform PC CMR just before aortic arch branching (for technical details see table 4). Analysis: Contours should be traced in all phases on the magnitude images using dedicated software. The presence of offset errors has been reported for scanners of all vendors, and may reduce the accuracy of the results [24]. Thus correction for offset errors is strongly recommended; this can be achieved by repeating measurements on a gel phantom or by measuring the signal of an area of similar size to the vessel of interest in nearby stationary tissue. Some newer post-processing software versions also offer an integrated flow correction algorithm.

\section{Myocardial stress perfusion}

Perfusion studies should be performed according to standard protocols with pulse sequences achieving the parameters given in table 5 [1, 2, 26, 27].

Contraindications must be carefully ruled out. Patients are prepared with absence of caffeine intake, etc. before the examination, as proposed by standard protocols (see also section 3 'Safety aspects').

Sequences: gradient echo, gradient echo-planar, SSFP. Typical parameters for a T1-weighted saturation-recovery gradient echo sequence for first-pass myocardial perfusion imaging are given in table 5 .

Vasoactive agents: adenosine $(140 \mu / \mathrm{kg} / \mathrm{min})$, dipyridamole.

After at least 3 minutes of vasodilator perfusion, a bolus of a GBCA $(0.05-0.1 \mathrm{mmol} / \mathrm{kg})$ at $5 \mathrm{ml} / \mathrm{s}$ is injected followed by a flush of saline $(25 \mathrm{ml}$ at $5 \mathrm{ml} / \mathrm{s}$ ) (children $10 \mathrm{ml}$ with $3 \mathrm{ml} / \mathrm{s}$ ).

Minimum three (adults three-five) short-axis slices are used to cover the left ventricle.

Scanning of 60 heartbeats after start of contrast agent infusion guarantees full coverage of the GBCA first pass during the breath-hold manoeuvre. 
Late gadolinium enhancement

Late gadolinium enhancement (LGE) imaging should be performed according to standardised protocols [1, 27]:

Available sequences include 2D segmented inversion recovery gradient echo pulse and phase-sensitive inversion recovery (PSIR) sequences. Parameters are shown in table 6 .

Image acquisition is started 10-20 minutes following injection of $0.1-0.2 \mathrm{mmol} / \mathrm{kg}$ of GBCA, in breath-hold if possible. Usually short-axis, two-, three- and fourchamber images are acquired, if necessary RV longaxis, inflow and outflow view, RVOT view.

Inversion time should be adjusted according to the contrast medium dose administered and time after injection.

In infants / small children, spatial resolution can be increased by reducing the size of the voxels and increasing signal averages for more signal. For adequate recovery between inversion pulses, acquisition of every third of four heart beats (instead of every other heart beat) should be considered. Image acquisition should be kept short by using a turbo factor to avoid blurring. Reporting: left ventricle 17 segment model, right ventricle 9 segment model $[28,29]$ (for examples see figs $4 \mathrm{a}$ and $4 \mathrm{~b})$.

\section{Reporting}

Bearing in mind that every centre uses its own report form, we recommend that a comprehensive report should include the aspects listed below.

\section{General}

In CHD, description of the segmental anatomy is useful. This includes cardiac situs, cardiac position, relationship of great arteries, pulmonary and systemic venous return, atrioventricular and ventriculoarterial relationships. Quantitative reference values can be found in Kawel-Boehm et al. [30].

\section{Ventricular volumetry}

End-diastolic and end-systolic ventricular volumes (absolute and indexed to body surface area), ejection

Table 6: Technical details late gadolinium enhancement.

\begin{tabular}{lll}
\hline & $<\mathbf{1 0} \mathbf{~ k g}$ & $\mathbf{1 0} \mathbf{~ k g}$ \\
\hline In-plane resolution $(\mathbf{m m})$ & $1.0-1.4$ & $1.3-1.7$ \\
\hline Slice thickness $(\mathbf{m m})$ & $6-8$ & $6-8$ \\
\hline Image acquisition timing & 3 RR or 4 RR & $2 \mathrm{RR}$ \\
\hline Trigger delay & Diastole or systole & Diastole \\
\hline
\end{tabular}

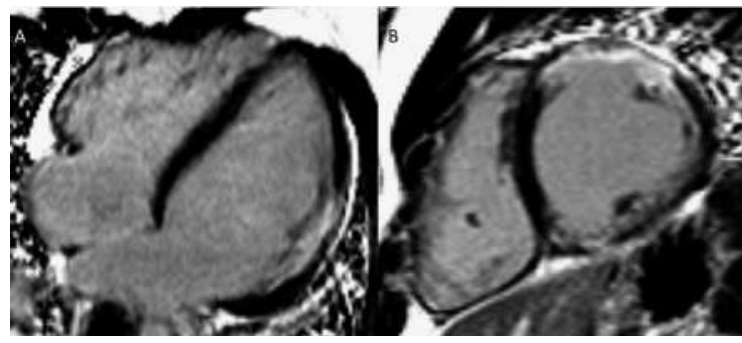

Figure 4a: Late gadolinium enhancement (LGE). 21-year-old patient with arterial switch for transposition of the great arteries (TGA) after postoperative myocardia ischaemia. Extensive area with subendocardial LGE in anterolateral segment (A) and in the inferior mid-ventricular segment (B).

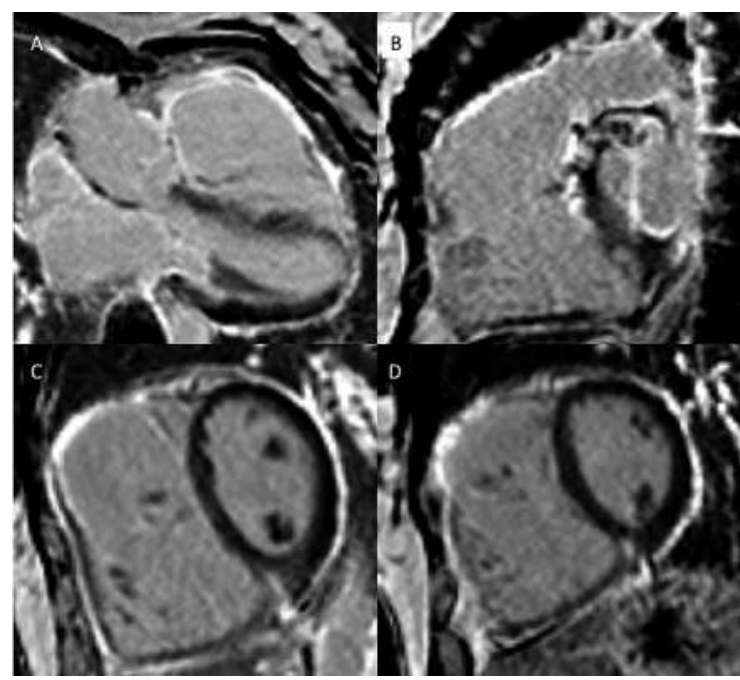

Figure 4b: Patient with transannular patch repair and VSD closure for tetralogy of Fallot and residual severe PR. LGE in RVOT aneurysm (A), LGE of the RVOT aneurysm and VSD patch area and inferior insertion point (RV hypertrophy) (B), LGE in the RV free wall (C-D).

fraction and, optionally, mass (absolute and indexed to body surface area)

\section{Flow by PC CMR}

Net forward flow, regurgitant fraction and volume if applicable; heart rate.

In the case of turbulent flow in the MPA, use of flow measurements in right and left pulmonary branch arteries or in each pulmonary vein to calculate pulmonary stroke volume is recommended. In the case of turbulent flow in the ascending aorta, PC CMR can be performed just before aortic arc branching.

In the case of significant valvular stenosis, maximum flow velocity can theoretically be used to calculate pressure gradients with the Bernoulli equation. However, echocardiography is clearly superior for quantification of stenosis and should be preferred to CMR. 


\section{Vascular dimensions}

Exact description of how and where measurements have been taken is important for reproducibility and comparability of the measurements in the follow-up studies. The quality of images, i.e., non-ECG-triggered CE MRA or ECG-triggered SSFP, must be specified. The aortic root should be visualised and measured with a gated sequence, such as SSFP, in order to avoid blurring due to the important vessel pulsatility.

Z-score calculators (validated for echocardiography): http://marfan.org/; http://www.parameterz.com/.

The following levels of measurement are recommended.

Aorta:

- Root: cusp to cusp, commissure to sinus, measured on SSFP or gradient echo, in systole in children, in diastole in adults [31].

- Sinotubular junction, ascending aorta at right pulmonary artery level, aortic arch, descending aorta at left pulmonary artery level, and at the level of the diaphragm, measured on CE MRA.

Pulmonary arteries:

- MPA, left pulmonary artery (LPA), right pulmonary artery (RPA) measured on CE MRA or 3D SSFP.

\section{Protocols for specific lesions}

At the end of every examination obtain noninvasively blood pressure in the right arm.

\section{1 Coarctation of the aorta, before and after repair}

\begin{tabular}{|c|c|}
\hline \multirow[t]{4}{*}{ Mandatory } & Ventricular volumetry \\
\hline & CE MRA or 3D SSFP of thoracic aorta \\
\hline & $\begin{array}{l}\text { PC CMR: } \\
\text { Ascending aorta, descending aorta at the } \\
\text { level of diaphragm }\end{array}$ \\
\hline & $\begin{array}{l}\text { LGE (at least once at age of } 16 \text { to } 20 \\
\text { years) }\end{array}$ \\
\hline \multirow[t]{4}{*}{ Optional } & Spin echo aortic arch long axis \\
\hline & $\begin{array}{l}\text { Cine CMR aortic root perpendicular to } \\
\text { vascular axis and aortic arch long axis }\end{array}$ \\
\hline & $\begin{array}{l}\text { PC CMR proximal and distal to coarcta- } \\
\text { tion and descending aorta for assess- } \\
\text { ment of collateral flow }\end{array}$ \\
\hline & LGE \\
\hline
\end{tabular}

Key reporting: location, dimensions, and severity of aortic obstruction; arch sidedness and branching order; arch dimensions, presence of aneurysm, dissection or collateral vessels, collateral flow to the descending aorta; LV volumes and systolic function, ventricular mass; aortic valve morphology, stenosis, and regurgitation (for examples see figs $2 \mathrm{~b}$ and 3 ).

\section{Aortic diseases (Marfan, Loeys-Dietz, familial aortic diseases)}

\begin{tabular}{ll}
\hline Mandatory & Ventricular volumetry (RV and LV) \\
\cline { 2 - 2 } & $\begin{array}{l}\text { Cine CMR: stack of orthogonal views of } \\
\text { aortic root, no gap, } 4-8 \mathrm{~mm} \text { slice thickness }\end{array}$ \\
& $\begin{array}{l}\text { CE MRA or 3D SSFP of thoracic and } \\
\text { abdominal aorta, pulmonary arteries }\end{array}$ \\
\hline PC CMR: ascending aorta \\
\hline Optional & Loeys-Dietz/Ehlers-Danlos syndrome and \\
& if applicable: CE MRA from head to iliac \\
bifurcation
\end{tabular}

Key reporting elements: description of aortic valve morphology, regurgitation, LV and RV volumes and systolic function, diameters of thoracic and abdominal aorta, ostial coronary arteries (especially after composite graft), signs of dissection, z-score calculators: http://marfan.org/; http://www.parameterz.com/

\section{D-transposition of great arteries \\ $D$-transposition of the great arteries following an arterial switch operation}

\begin{tabular}{ll}
\hline Mandatory & Ventricular volumetry (RV and LV) \\
\cline { 2 - 2 } & Cine CMR: long-axis RVOT, LVOT, long-axis \\
RV and LV & CE MRA or 3D SSFP of thoracic aorta, \\
& pulmonary arteries \\
\hline PC CMR: ascending aorta, MPA, LPA, RPA \\
\hline Sptional & Stress myocardial perfusion and LGE \\
\hline Cine CMR: long-axis MPA, LPA, RPA \\
\hline 3D SSFP ostial and proximal coronary \\
arteries, PAs, thoracic aorta
\end{tabular}

Key reporting elements: location and severity of ascending aorta, MPA and branch PA obstruction; branch PA flow distribution; proximal coronary artery course; aortic root dilation; aortic and pulmonary regurgitation; RV and LV volumes and systolic function; myocardial perfusion (if performed)

\section{$D$-transposition of the great arteries following an atrial switch operation}

\begin{tabular}{ll}
\hline Optional & Ventricular volumetry (RV and LV) \\
\cline { 2 - 2 } & Cine CMR: long-axis RVOT, LVOT, long-axis \\
SVC and IVC baffle & CE MRA or 3D SSFP of systemic and \\
pulmonary venous return, thoracic aorta, \\
pulmonary trunk and arteries
\end{tabular}


Key reporting elements: location and severity of systemic and pulmonary venous pathway obstruction, baffle leak, LV and RV volumes and systolic function, severity and mechanism of left or right ventricular outflow tract obstruction, tricuspid regurgitation, ratio of pulmonary to systemic blood flow (Qp/Qs).

\section{Congenitally corrected transposition of the great arteries}

\begin{tabular}{ll}
\hline Mandatory & Ventricular volumetry (RV and LV) \\
\cline { 2 - 2 } & \begin{tabular}{l} 
Cine CMR: long-axis RVOT, LV and RV \\
\hline PC CMR: ascending aorta, MPA
\end{tabular} \\
\hline & Cine CMR axial stack \\
\hline & CE MRA / 3D SSFP of all intrathoracic \\
& Messels \\
\hline
\end{tabular}

Key reporting elements: location and severity of subpulmonary obstruction, LV and RV volumes and systolic function, tricuspid regurgitation, Qp/Qs.

\section{Ebstein's anomaly of the tricuspid valve}

\begin{tabular}{ll}
\hline Mandatory & Ventricular volumetry (RV and LV) \\
\cline { 2 - 2 } & $\begin{array}{l}\text { Cine CMR: long-axis RVOT, LV and RV } \\
\text { Cine CMR: 3- and 4-chamber view tricus- } \\
\text { pid valve }\end{array}$ \\
\hline & Cine CMR axial stack \\
\hline PC-CMR: ascending aorta, MPA \\
\hline Optional & CE MRA / 3D SSFP pulmonary arteries \\
& Cind aorta \\
\hline
\end{tabular}

Key reporting elements: description of tricuspid valve morphology, apical displacement of the septal leaflet (in $\mathrm{mm}$ ), tethering of the anterior leaflet, severity of tricuspid regurgitation, pulmonary stenosis, LV and RV volumes and systolic function, presence of an atrial septal defect, Qp/Qs based on flow measurements.

\section{Tetraology of Fallot, pulmonary atresia with ventricular septal defect, truncus arteriosus following complete repair, surgical correction with RV-PA conduits}

\begin{tabular}{ll}
\hline Mandatory & $\begin{array}{l}\text { Ventricular volumetry (RV and LV) } \\
\text { Cine CMR: long-axis RVOT stack in double } \\
\text { oblique sagittal plane (especially before } \\
\text { percutaneous pulmonary valve implanta- } \\
\text { tion) }\end{array}$ \\
& $\begin{array}{l}\text { CE MRA / 3D SSFP of thoracic vasculature } \\
\text { and proximal coronary arteries }\end{array}$ \\
\hline PC CMR: ascending aorta, MPA / RV-PA \\
conduit
\end{tabular}

\begin{tabular}{|c|c|}
\hline \multirow[t]{3}{*}{ Optional } & $\begin{array}{l}\text { PC CMR: RPA, LPA, AV valves, SVC, } \\
\text { descending aorta at diaphragm }\end{array}$ \\
\hline & LGE \\
\hline & $\begin{array}{l}\text { Cine CMR: long-axis RVOT stack in double } \\
\text { oblique axial plane (before percutaneous } \\
\text { pulmonary valve implantation) }\end{array}$ \\
\hline
\end{tabular}

Key reporting elements: RV and LV volumes and systolic function (include RV infundibular aneurysms, if present, in RV volumes), RVOT and pulmonary arterial anatomy, quantification of pulmonary regurgitant volume and fraction, branch pulmonary artery flow distribution, Qp/Qs, proximal coronary artery anatomy for percutaneous pulmonary valve implantation.

\section{Partial anomalous pulmonary venous connec- tion/sinus venosus defect}

\begin{tabular}{|c|c|}
\hline \multirow[t]{4}{*}{ Mandatory } & Ventricular volumetry (RV and LV) \\
\hline & $\begin{array}{l}\text { Cine CMR: axial stack covering all pulmo- } \\
\text { nary venous connections, oblique stack } \\
\text { covering anomalous veins, sinus venous } \\
\text { defect }\end{array}$ \\
\hline & $\begin{array}{l}\text { CE MRA / 3D SSFP of pulmonary venous } \\
\text { return }\end{array}$ \\
\hline & PC CMR: ascending aorta, MPA \\
\hline \multirow[t]{2}{*}{ Optional } & $\begin{array}{l}\text { PC CMR: RPA, LPA, pulmonary veins, SVC } \\
\text { above and below anomalous pulmonary } \\
\text { veins }\end{array}$ \\
\hline & $\begin{array}{l}\text { Cine CMR: oblique coronal stack covering } \\
\text { anomalous veins/sinus venosus defect }\end{array}$ \\
\hline
\end{tabular}

Key reporting elements: RV and LV volumes and systolic function, connections and drainage of the pulmonary veins, size of the sinus venosus defect, Qp/Qs (for examples see figs 5 and 6 ).

\section{Functionally single ventricle after bidirectional cavopulmonary connection (BCPC)}

\begin{tabular}{ll}
\hline Mandatory & $\begin{array}{l}\text { Ventricular volumetry (including all vol- } \\
\text { umes contributing to systemic output) }\end{array}$ \\
\cline { 2 - 2 } Cine CMR: \\
Axial stack covering the entire thorax \\
$\begin{array}{l}\text { Aortic arch in long axis if status after arch } \\
\text { enlargement/coarctation repair }\end{array}$ \\
\hline CE MRA/3D SSFP of thoracic vasculature \\
\hline PC CMR: ascending aorta, SVC, RPA, LPA, \\
pulmonary veins, descending aorta at \\
diaphragm, decompressing veins
\end{tabular}

Key reporting elements: ventricular volumes and systolic function (including all volumes contributing to systemic output, exclude interventricular septum if 


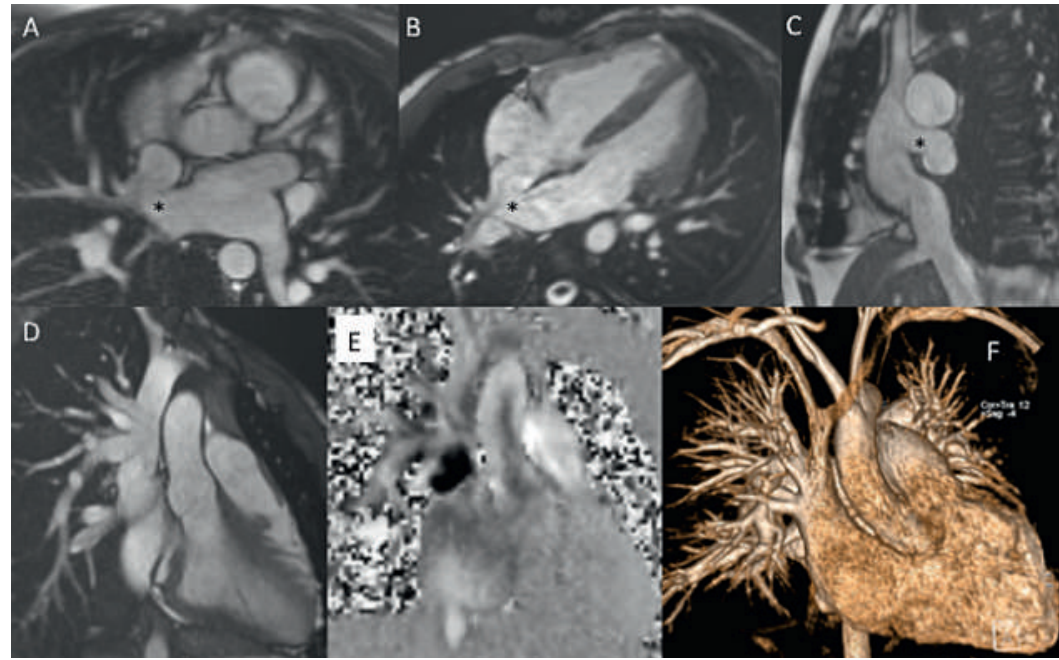

Figure 5: Uncorrected sinus venosus defect from SVC-type with partial anomalous pulmonary venous drainage (PAPVD) of the right upper pulmonary vein.

SSFP cine still frames in axial, oblique and sagittal orientation (A-C), SVC with PAPVD in SSFP cine still frame (D), PC CMR orthogonal en face view of the defect and L-R shunt (black area) (E), CE MRA angiography with visualisation of the the PAPVD (F).

* Sinus venosus defect.

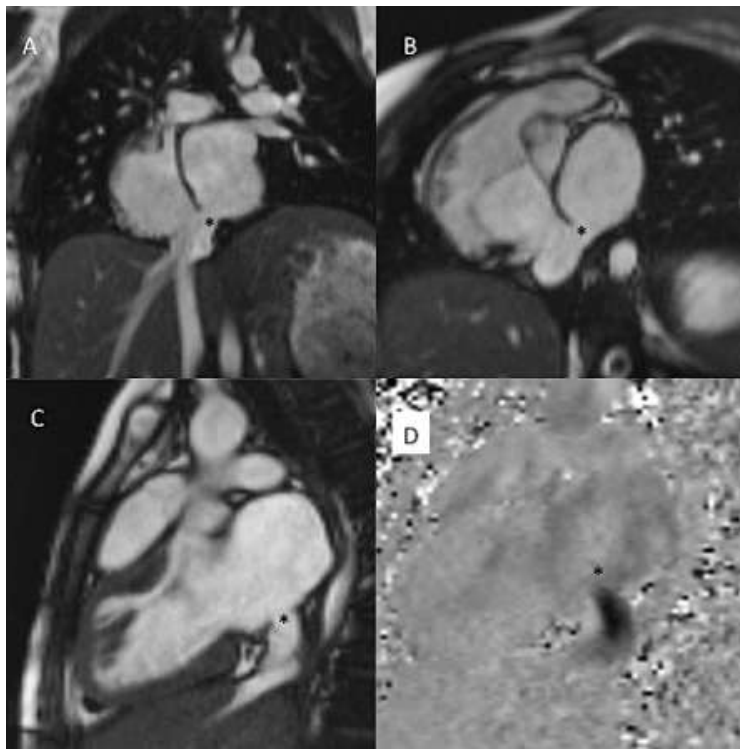

Figure 6: Sinus venosus defect from IVC-type. SSFP cine still frame in coronal, oblique and sagittal views (A-C), inplane PC CMR with black signal of the L-R shunt.

* Sinus venosus defect.

Correspondence:

Dr. Tobias Rutz

Division of Cardiology and Cardiac MR Center

Centre Hospitalier Universitaire Vaudois

Rue du Bugnon 46

CH-1011 Lausanne

tobias.rutz[at]chuv.ch

, BCPC and pulmonary artery obstruc branch pulmonary artery flow distribution, aortic arch obstruction, systemic-pulmonary collateral arteries and blood flow, Qp/Qs, veno-venous collaterals.

\section{Borderline LV hypoplasia before surgery}

\begin{tabular}{ll}
\hline Mandatory & $\begin{array}{l}\text { Ventricular volumetry (no gap, thinnest } \\
\text { possible slice, consider gradient echo) }\end{array}$ \\
\cline { 2 - 2 } $\begin{array}{l}\text { Cine CMR: LVOT long axis, aortic arch } \\
\text { in long axis }\end{array}$ & $\begin{array}{l}\text { CE MRA / 3D SSFP of thoracic vasculature } \\
\text { PC CMR: ascending aorta }\end{array}$ \\
\hline LGE (endocardial fibroelastosis) \\
\hline Optional & PC CMR: SVC, RPA, LPA, PDA, descending \\
& aorta at diaphragm, AV valves
\end{tabular}

Key reporting elements: LV volumes and systolic function, LV inlet and outlet anatomy, aortic arch and PDA anatomy, Qp/Qs, endocardial fibroelastosis.

\section{Fontan circulation}

\begin{tabular}{|c|c|}
\hline \multirow[t]{4}{*}{ Mandatory } & Ventricular volumetry \\
\hline & CE MRA / 3D SSFP of thoracic vasculature \\
\hline & PC CMR: ascending aorta, MPA if present \\
\hline & $\begin{array}{l}\text { PC CMR (free breathing): branch PAs, SVC, } \\
\text { IVC }\end{array}$ \\
\hline \multirow[t]{5}{*}{ Optional } & $\begin{array}{l}\text { Cine CMR: coronal/oblique stack of Fontan } \\
\text { baffles and anastomoses in long axis }\end{array}$ \\
\hline & $\begin{array}{l}\text { Cine CMR: long-axis LV and RV, LVOT and } \\
\text { RVOT, aortic arch if after aortic arch en- } \\
\text { largement/coarctation repair }\end{array}$ \\
\hline & LGE \\
\hline & $\begin{array}{l}\text { PC CMR: AV valves, pulmonary veins, } \\
\text { aortic arch if after aortic arch enlarge- } \\
\text { ment /coarctation repair }\end{array}$ \\
\hline & Fibrosis imaging of liver \\
\hline
\end{tabular}

Key reporting elements: Size and function of ventricular volumes contributing to systemic output (exclude interventricular septum if applicable), Fontan pathway, SVC, IVC, branch PAs, pulmonary vein, and aortic arch obstruction; Fontan baffle defects; ventricular parameters; valve regurgitation; aortopulmonary or pulmonary veno-venous collaterals, Qp/Qs.

\section{Disclosure statement}

No financial support and no other potential conflict of interest relevant to this article was reported.

\section{Acknowledgements}

The group of authors would like to thank the presidents of the working groups and societies who endorsed this manuscript for the support and help during the preparation of the manuscript: Dr. Judith Bouchardy, president of the working group "Adult Congenital Heart Disease (WATCH)" of the Swiss Society of Cardiology; PD Dr. Hajo Müller, president of the working group "Echocardiography and Cardiac Imaging" of the Swiss Society of Cardiology; PD Dr. Christian Balmer, president of the Swiss Society of Paediatric Cardiology; Prof. Jens Bremerich, president of the "Ressort Cardiac Imaging" of the Swiss Society of Radiology" (also co-author of the document).

\section{References}

The full list of references is included in the online version of the article at www.cardiovascmed.ch. 